\title{
Robot Experiment of Torque Learning for Biped Balance with respect to Periodic External Force
}

\author{
Satoshi Ito \\ Fac. of Eng./ BMC Research Center \\ Gifu Univ./RIKEN \\ Gifu/Nagoya, Japan \\ Email: satoshi@cc.gifu-u.ac.jp \\ Haruhisa Kawasaki \\ Fac. of Eng. \\ Gifu Univ. Gifu, Japan \\ Email: h_kawasa@cc.gifu-u.ac.jp
}

\author{
Kohei Moriki \\ Graduate School of Eng. \\ Gifu Univ. \\ Gifu, Japan \\ Email: j3132047@guedu.cc.gifu-u.ac.jp \\ Minoru Sasaki \\ Fac. of Eng. \\ Gifu Univ. Gifu, Japan \\ Email: sasaki@cc.gifu-u.ac.jp
}

\begin{abstract}
In this paper, we report robot experimental results of balance control and torque pattern learning under the influence of periodic external force. In the previous paper, we propose not only a control method for keeping balance based on the feedback of ground reaction forces but also a learning method of torque pattern for balancing in such a stationary environment as a periodic external force is exerted. In order to maintain the balance, the feedback of ground reaction forces is essential. As a result of learning, however, the information on them become unnecessary because the torque pattern for balancing is stored in the controller and thus the balancing is achieved in a feedforward manner. Here, we aim at verifying this control and learning scheme by experiments with a simple robot.
\end{abstract}

\section{INTRODUCTION}

One of problems in the recent robotics is how to make robots behave even under uncertain environmental conditions. When designers or users plan behaviors of the robots to achieve some intended tasks, they implicitly assume some conditions of environment, under which they calculate reference trajectories, typically of joint angles. In many practical cases, however, actual environmental conditions are not the same as the assumed ones at the planning stage of motions. To absorb such differences, feedback control is implemented in practical robot control.

The feedback control is an efficient method for truly realizing the designed reference trajectories of joint angles. However, the reference trajectories for feedback control are not always appropriate for the current environment of the robot. In case of animals, they prepare some alternative motion patterns and choose an appropriate one among them. This implies that, in such animal motions, some reference trajectories have already stored as motion patterns. In this effective motor control strategy, however, there are some questions to be answered: How do motion patterns are firstly produced to match well the environmental conditions? What conditions are necessary for producing the motion patterns? Or, what in form are they memorized to the controllers or the motion pattern generators? To discuss these problems, we give the following assumptions: The environmental conditions are steady. The steady environment possess some regularities that should yield patterns in motions. And, the motion patterns are represented as profiles of the joint torque. Certainly, they contain important information on how much the torque should be output at what timing. Based on these assumptions, the learning process of joint torque profile is treated as the storage of the motion pattern. In the early stage of learning process, such torque pattern will be depending on the sensory feedback due to the uncertainties in environment. After a while, however, the regularity in the environment is learned through the repetitive motion in the steady condition. Based on this regularity, the torque pattern is internally produced without the sensory feedback and finally generated mainly in a feedforward manner.

Following the above scenario, we proposed a control and learning scheme of motion [1], [2], [3]. There, we selected the static balance control of biped system as an objective motion, since its dynamics can be described in the simple equations. In addition, regardless of the simple dynamics, the biped balance is an elaborate motion that only a few kinds of animals, like humans, can achieve, and is a fundamental motion for performing the biped human actions. To understand this control mechanism is therefore important for realizing the legged motion in the real world containing many uncertain factors. The steady condition of the environment was expressed as unknown periodic external forces with known period exerted to the balancing system. The purpose of this problem setting was to maintain the biped balance against the unknown external force and finally to acquire an environmentdependent torque pattern for balancing. For this problem, we proposed a control method that achieves the balance control in unknown environment by introducing the feedback of ground reaction forces, and a learning rule of the torque profile by estimating the exerted external forces. In this paper, we report the experimental verification of this formulation using a simple robot. 


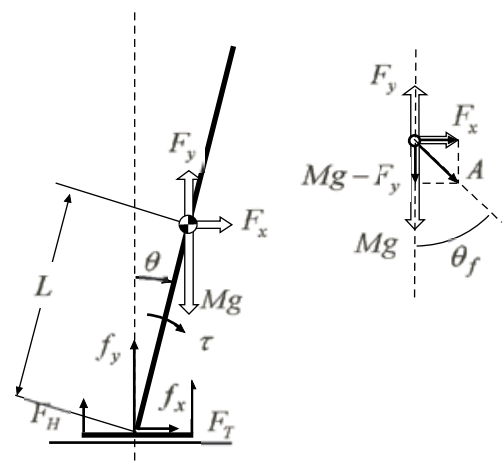

Fig. 1. Simple model of biped balance control.

\section{THEORIES}

\section{A. A simple model of biped balance}

Humans mainly use their ankle joints when small disturbance is applied [4]. Based on this observation, the biped behavior in the static balance is sufficiently expressed as a simple model consisting of a body segment and a foot segment, as illustrated in Fig. 1. To make the analysis simple, the motion within the sagittal plane is considered. Two links are connected at the ankle joint, which is located at the center of the foot segment symmetrical to the anterior-posterior direction and as low as the ground surface. Thus, the posture is represented only by the angles of the ankle joint. The angle and angular velocity of the ankle joint are assumed to be detectable, and the balance is controlled using the ankle joint torque. The foot segment contacts the ground at two points only, i.e., the toe and the heel, and they are always in contact to the ground owing to the balance control. The vertical components of ground reaction forces $F_{T}$ (at the toe) and $F_{H}$ (at the heel) are assumed to be detectable. The friction between the foot segment and the ground is so large that the foot segment does not slip on it. To this simple model, an unknown external force is exerted, whose horizontal and vertical components are $F_{x}$ and $F_{y}$, respectively. This external force is unknown and represents the conditions of the environment.

The dynamics of the body segment is represented simply by the motion equation of the inverted pendulum,

$$
I \ddot{\theta}=M L g \sin \theta+F_{x} L \cos \theta-F_{y} L \sin \theta+\tau,
$$

where $M$ is the mass of the body part, $I$ is the inertial moment of the body part around the ankle joint, $L$ is the length between the ankle joint and the COG of the body part, $\theta$ is the ankle joint angle from the vertical direction, $\tau$ is the ankle joint torque, and $g$ is the gravitational acceleration. The foot segment, on the other hand, neither moves nor rotates if the balance is maintained. Then, a static relation of the exerted forces is obtained: using the balance of moment around the heel and toe, the ground reaction forces, $F_{T}$ and $F_{H}$, are described as

$$
\begin{gathered}
F_{T}=\left(-\tau / \ell+m g+f_{y}\right) / 2, \\
F_{H}=\left(\tau / \ell+m g+f_{y}\right) / 2,
\end{gathered}
$$

where $m$ is the total mass of the foot, $\ell$ represents the distance from the ankle joint to the toe or heel, and, $f_{y}$, the force from the body segments, is described as

$$
f_{y}=-M L \ddot{\theta} \sin \theta-M L \dot{\theta}^{2} \cos \theta+M g-F_{y} .
$$

From (2) and (3), we can obtain the relation between $F_{H}-F_{T}$ and $\tau$,

$$
F_{H}-F_{T}=\tau / \ell
$$

For convenience of calculation, we transform the motion equation (1) as follows:

$$
\begin{aligned}
I \ddot{\theta} & =\left(M g-F_{y}\right) L \sin \theta+F_{x} L \cos \theta+\tau \\
& =A L \sin \left(\theta-\theta_{f}\right)+\tau
\end{aligned}
$$

where

$$
A=\sqrt{\left(M g-F_{y}\right)^{2}+F_{x}^{2}}
$$

and $\theta_{f}$, as shown in Fig. 1, is a constant that satisfies these equations,

$$
\sin \theta_{f}=-F_{x} / A, \quad \cos \theta_{f}=\left(M g-F_{y}\right) / A .
$$

\section{B. Balance control in uncertain environment}

1) Control with ground reaction force feedback: In order to maintain body balance, both $F_{T}$ and $F_{H}$ must be kept positive. Furthermore, the stability margin [5] will be greatest when body mass is evenly distributed between the toe and the heel. Thus, the goal of balance control here is to converge $F_{H}-F_{T}$ to zero without allowing the body segment to fall.

According to (5), if we define the ankle joint torque as

$$
\tau=-K_{I} \int\left(F_{H}-F_{T}\right) d t
$$

then $F_{H}-F_{T}$ will certainly converge to zero. However, this control law does not result in the maintenance of an upright posture. For example, assume here that the body segment leans slightly to the toe side. This leads the weight to be distributed more to the toe than to the heel, i.e., $F_{T}>F_{H}$. If the positive torque is generated according to (9), the body segment lean more to the toe side and will consequently tumble to this side.

To avoid such situation, we combine with it the PD (proportional and derivative) control, which stabilizes the upright posture. The control law we propose here is

$$
\tau=-K_{d} \dot{\theta}-K_{p} \theta+K_{f} \int\left(F_{H}-F_{T}\right) d t .
$$

Here, $K_{d}, K_{p}$, and $K_{f}$ are feedback gains.

2) Stationary state: To analyze the dynamics as a result of the control law (10), we introduce a new state variable $\tau_{f}$ which is defined as

$$
\tau_{f}=\int\left(F_{H}-F_{T}\right) d t
$$

Then, (10) becomes

$$
\tau=-K_{d} \dot{\theta}-K_{p} \theta+K_{f} \tau_{f} .
$$

Substituting it for (6), we obtain

$$
\ddot{\theta}=A L \sin \left(\theta-\theta_{f}\right)-K_{d} \dot{\theta}-K_{p} \theta+K_{f} \tau_{f} .
$$




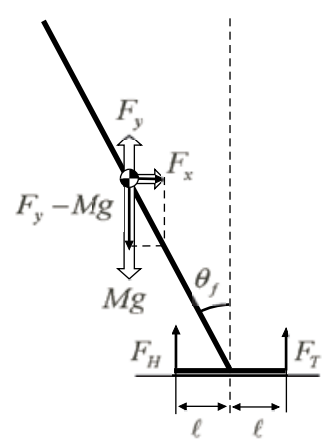

Fig. 2. Stationary posture by proposed control law.

On the other hand, differentiating (11) and next using (5) and (12), we obtain

$$
\dot{\tau}_{f}=\left(-K_{d} \dot{\theta}-K_{p} \theta+K_{f} \tau_{f}\right) / \ell .
$$

The stationary state is calculated by putting $\ddot{\theta}=\dot{\theta}=0$ and $\dot{\tau}_{f}=0$. Most importantly, $F_{H}-F_{T}=0$ is certainly achieved by (10), since $F_{H}-F_{T} \equiv \dot{\tau}_{f}=0$. On the other hand, the stationary posture is obtained by solving the following two algebraic equations

$$
\begin{gathered}
A L \sin \left(\theta-\theta_{f}\right)-K_{p} \theta+K_{f} \tau_{f}=0, \\
\left(-K_{p} \theta+K_{f} \tau_{f}\right) / \ell=0 .
\end{gathered}
$$

As a result, $\theta=\theta_{f}$ is satisfied at the stationary state. This implies that the stationary posture adaptively changes with the environmental conditions, since $\theta_{f}$ depends on the unknown external forces $F_{x}$ and $F_{y}$. At this posture, the body segment takes the direction of the force resulting from the gravitational and external forces, as shown in Fig. 2. In this posture, the moments generated by these two forces cancel out each other around the ankle joint. Therefore, the ankle joint requires little torque or, theoretically, none at all.

3) Stability analysis: To examine the stability of this stationary state, we regard $\theta, \dot{\theta}$, and $\tau_{f}$ as state variables, and we linearize the differential equations around the equilibrium point, i.e., $\theta=\theta_{f}$ and $\tau_{f}=K_{p} \theta_{f} / K_{f}$. The linear differential equation is

$$
\left[\begin{array}{c}
\dot{\theta}_{1} \\
\dot{\theta}_{2} \\
\dot{\tau}_{f}
\end{array}\right]=\left[\begin{array}{ccc}
0 & 1 & 0 \\
\frac{A L-K_{p}}{I} & -\frac{K_{d}}{I} & \frac{K_{f}}{I} \\
-\frac{K_{p}}{\ell} & -\frac{K_{d}}{\ell} & \frac{K_{f}}{\ell}
\end{array}\right]\left[\begin{array}{c}
\theta_{1} \\
\theta_{2} \\
\tau_{f}
\end{array}\right]
$$

where $\theta_{1}=\theta$ and $\theta_{2}=\dot{\theta}$. The characteristic equation of this linear system is given by

$$
\lambda^{3}+p_{2} \lambda^{2}+p_{1} \lambda+p_{0}=0
$$

where

$$
p_{2}=\frac{K_{d} \ell-K_{f} I}{I \ell}, p_{1}=\frac{K_{p}-A L}{I}, p_{0}=\frac{K_{f} A L}{I \ell}
$$

According to Routh/Hurwitz criterion, the necessary and sufficient conditions to stabilize the equilibrium point are given as

$$
p_{0}>0, p_{1}>0, p_{2}>0, p_{1} p_{2}-p_{0}>0
$$

From these inequalities, we can derive the following conditions:

$$
\begin{gathered}
K_{p}>A L>0 \\
\frac{\ell}{I} K_{d}>K_{f}>0 \\
\left(K_{d} \ell-K_{f} I\right) K_{p}>K_{d} \ell A L
\end{gathered}
$$

In summary, if the feedback gains are set so that (21)(21) hold, the stationary posture in Fig. 2 becomes locally asymptotically stable.

\section{Torque pattern learning from regularity in environment}

1) Construction of ankle joint torque: The control law in the previous section achieves static balance even if unknown factors, which was represented by unknown external forces, exist in the environmental conditions. A feature of this control law is found in the feedback on ground reaction forces. However, once the stationary state is achieved, the adequate posture in the current environment (which was unknown) becomes known from the stationary posture. If this posture is memorized in the controller, balance is maintained only by positional control without feedback of ground reaction forces that was essential in an uncertain environment. Namely, the feedback information of ground reaction forces is utilized to obtain the adequate posture in a steady unknown environment.

Now, we extend a steady environmental conditions from static, i.e., constant external force, to periodic with known period $T_{e}$. The purpose here is to compose a control law for balancing under periodic external forces and a learning law that reduces the usage of information on ground reaction forces. For this purpose, we construct an ankle joint torque from two terms as

$$
\tau=[F . F]+\left[-K_{d} \dot{\theta}-K_{p} \theta+K_{f} \int\left(F_{H}-F_{T}\right) d t\right]
$$

The second term including the feedback on the ground reaction force is the same as (10), which works to cope with unknown factors in environmental conditions, while the first term compensates the periodic external forces in a feedforward manner without ground reaction force feedback. We will compose a learning rule such that the second term gradually decreases.

We construct the feedforward term by estimating the periodic external force. Here, we assumed that the period of the periodic external force $T_{e}$ is known. Then, the external force is expanded to a Fourier series

$$
\begin{aligned}
& F_{x}=\sum_{k}^{n}\left(\alpha_{k}^{(x)} S_{k}+\beta_{k}^{(x)} C_{k}\right) \\
& F_{y}=\sum_{k}^{n}\left(\alpha_{k}^{(y)} S_{k}+\beta_{k}^{(y)} C_{k}\right)
\end{aligned}
$$


where, $S_{k}=\sin k \omega_{e} t, C_{k}=\cos k \omega_{e} t, \omega_{e}=2 \pi / T_{e}$. Substituting (1) by (25) and (26), we obtain

$$
\begin{array}{r}
I \ddot{\theta}-M L g S-\sum_{k}^{n}\left(\alpha_{k}^{(x)} S_{k}+\beta_{k}^{(x)} C_{k}\right) L C \\
+\sum_{k}^{n}\left(\alpha_{k}^{(y)} S_{k}+\beta_{k}^{(y)} C_{k}\right) L S=\tau,
\end{array}
$$

where $C=\cos \theta$ and $S=\sin \theta$. The left-hand side can be written in a linear form for unknown parameters as

$$
\begin{gathered}
Y \sigma=\tau \\
Y=\left[\ddot{\theta}, S, S_{0} C, C_{0} C, S_{0} S, C_{0} S\right. \\
\left.\cdots, S_{n} C, C_{n} C, S_{n} S, C_{n} S\right] \\
\sigma=\left[I,-M g L,-L \alpha_{0}^{(x)},-L \beta_{0}^{(x)}, L \alpha_{0}^{(y)}, L \beta_{0}^{(y)},\right. \\
\left.\cdots,-L \alpha_{n}^{(x)},-L \beta_{n}^{(x)}, L \alpha_{n}^{(y)}, L \beta_{n}^{(y)}\right]^{T} .
\end{gathered}
$$

These unknown parameters are estimated in the learning process.

2) Control and learning method: We here introduce a new unknown parameter $\phi$ based on $\sigma$

$$
\begin{gathered}
\phi=K_{I} \sigma, \\
K_{I}=\frac{K_{d} \ell}{K_{d} \ell-K_{f} I} .
\end{gathered}
$$

Using the estimated value of this parameter, i.e., $\hat{\phi}$, we define a control law as

$$
\begin{gathered}
\tau=Y_{r} \hat{\phi}-K_{d} s, \\
Y_{r}=\left[\ddot{\theta}_{r}, S, S_{0} C, C_{0} C, S_{0} S, C_{0} S,\right. \\
\left.\cdots, S_{n} C, C_{n} C, S_{n} S, C_{n} S\right]^{T}, \\
\dot{\theta}_{r}=-\frac{K_{p}}{K_{d}} \theta, \\
s=\dot{\theta}-\dot{\theta}_{r}-\frac{K_{f}}{K_{d}} \tau_{f} .
\end{gathered}
$$

In addition, we define a learning law of $\hat{\phi}$ as

$$
\dot{\hat{\phi}}=-\Gamma Y_{r}^{T} s
$$

where, $\Gamma$ is a positive definite diagonal matrix. Note that the first term does not contain feedback information on the ground reaction forces, and that the second term $-K_{d} s$ is the same as the right-hand side of (24).
3) Brief of behavioral analysis: Firstly, we set $\hat{\phi}(0)=0$. It implies that the feedforward torque is zero in the initial state and all the control torque are computed from the feedback information on the ground reaction forces. Under this initial condition, we show that the feedback torque decreases to zero according to learning, and finally the feedback torque is copied to the feedforward torque.

To begin with, we examine the decrement of the feedback torque. Consider the following function as a candidate Lyapunov function:

$$
V=\frac{1}{2} K_{I} I s^{2}+\frac{1}{2} \bar{\phi}^{T} \Gamma^{-1} \bar{\phi}(\geq 0)
$$

where $\bar{\phi}=\hat{\phi}-\phi$. Note that $K_{I}>0$ from (21)-(23). Differentiating (38), we obtain

$$
\dot{V}=K_{I} I s \dot{s}+\dot{\hat{\phi}}^{T} \Gamma^{-1} \bar{\phi} .
$$

Here, we use $\dot{\bar{\phi}}=\dot{\hat{\phi}}$ since $\phi$ is constant. After simple calculations, $\dot{V}$ becomes,

$$
\begin{aligned}
\dot{V} & =s Y_{r} \bar{\phi}-K_{d} s^{2}+\bar{\phi}^{T} \Gamma^{-1} \dot{\hat{\phi}} \\
& =\bar{\phi}^{T}\left(Y_{r}^{T} s+\Gamma^{-1} \dot{\hat{\phi}}\right)-K_{d} s^{2} .
\end{aligned}
$$

Then, substituting the above equation by (37), we finally obtain

$$
\dot{V}=-K_{d} s^{2} \leq 0
$$

Further differentiation by time produces the second derivative,

$$
\ddot{V}=-2 K_{d} s \dot{s} .
$$

We can prove the boundedness of $\ddot{V}$ if external force is bounded and differentiable. Using the Lyapunov-like lemma [6], we conclude that $\dot{V}$ converges to 0 and thus $s \rightarrow 0$, implying that the feedback torque becomes zero after learning.

Next, we discuss the effect of the learning on the torque profile. As the change of ankle joint originates from the external force, we here consider the transfer function from the external force to ankle joint angle. Here, we put the transfer function before learning $H_{b}(p)$. This transfer function can be calculated from (1), (5) and (10) after linearization around upright posture:

$$
H_{b}(p)=\frac{K_{f}\left(p^{2} I-M g L\right)\left(1-p \frac{\ell}{K_{f}}\right)}{p \ell\left(p K_{d}+K_{p}\right)} .
$$

Here, $p$ is the differential operator. On the other hand, we put the transfer function after learning $H_{a}(p)$. After learning, a new constraint $s=0$ is created as shown in the former part of this section. Accordingly, the transfer function is obtained from (1), (5) and the next equation,

$$
-K_{d} \dot{\theta}-K_{p} \theta+K_{f} \tau_{f}=0
$$

After all, $H_{a}(p)$ becomes

$$
H_{a}(p)=\frac{K_{f}\left(p^{2} I-M g L\right)}{p \ell\left(p K_{d}+K_{p}\right)}
$$




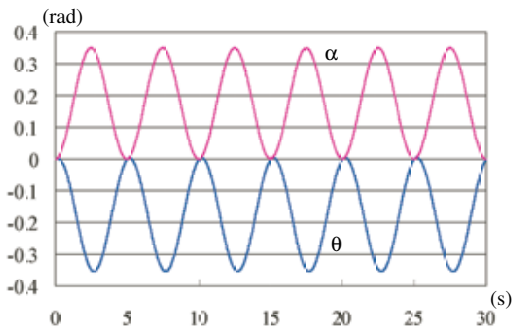

(a) Slope and ankle joint angles.

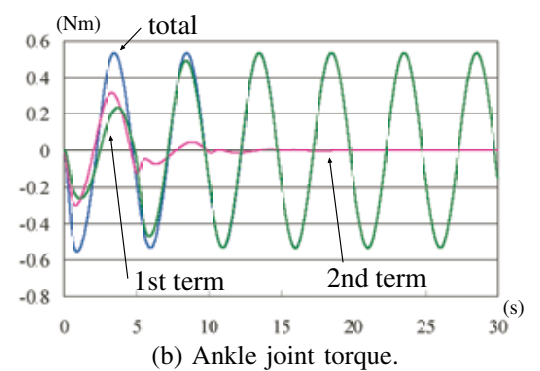

Fig. 3. Results from the proposed method.

Comparing $H_{b}(p)$ and $H_{a}(p)$, we can conclude that the profiles of the torque pattern before and after learning can be regarded as the same, if the condition $K_{f} \gg \ell$ is satisfied.

In the above analysis, however, the changes of torque pattern during the learning cannot be discussed. This analysis is difficult due to its high-nonlinearity. Thus, we will confirm it by computer simulations, as shown in the next section. If it can be confirmed, we can say that the feedback torque will be copied to the feedforward torque through the learning that estimates the periodic external force.

\section{Simulation}

We perform computer simulations to observe the learning effect. In computer simulations, we use a two-link model as illustrated in Fig. 2. The parameters are set as follows: $M=$ $2(\mathrm{~kg}), L=0.5(\mathrm{~m}), \ell=0.05(\mathrm{~m}), I=5 M L^{2} / 4\left(\mathrm{kgm}^{2}\right), K_{d}=$ $500, K_{p}=1000, K_{f}=25, \Gamma=\operatorname{diag}[100, \cdots, 100], n=10$. We define the periodic external force as

$$
\begin{gathered}
F_{x}=M g L \sin \alpha \\
F_{y}=M g L(1-\cos \alpha) \\
\alpha=\frac{\pi}{18}\left(1-\cos 2 \pi f_{e} t\right),
\end{gathered}
$$

where we set $f_{e}=0.2(\mathrm{~Hz})$, i.e., the period of the external force becomes $5(\mathrm{sec})$. This external force equivalently expresses the gravitational effect on a slope whose gradient is $\alpha$.

As shown in Fig. 3(a), the ankle joint angles are adjusted according to the periodic external forces. Furthermore, as shown in Fig. 3(b), the components of ankle joint torque vary so that the feedback term of the ground reaction forces decreases, while the feedforward term increases to occupy almost all of the total ankle joint torque.

\section{EXPERIMENTS}

\section{A. Setup}

Robot experiments were executed to examine the validity of theoretical analysis on torque pattern learning. In the experiment, we use a simple robot consisting of body and foot segment, as shown in Fig. 4(a). The ankle joint is actuated by DC servo motor (maxon RE25 20W) with 53:1 reduction gear. The angle of ankle joint is detected using a rotary encoder, and the angular velocity of it is calculated using the digital differential filter whose cut-off frequency is $10 \mathrm{~Hz}$. On the other hand, ground reaction forces are measured by four load cells attached on each corner of the sole, as shown in Fig. 4(b). The data measured in two front load cells are added to obtain the value of $F_{T}$, while those in two back load cells are used for $F_{H}$.

To change environmental conditions, we designed an active slope stand. The object of experiments is to make the robot stand erectly on it without tumbling. The slope is actuated by a DC servo motor (maxon RE25 20W) with the 370:1 reduction gear. The gradient of the slope is detected by rotary encoder installed to it.

\section{B. Conditions}

To apply periodical external forces to the robot, the gradient of the slope stand was altered from -0.12 (rad) to +0.12 (rad) periodically with the frequency $f_{e}=\omega_{e} / 2 \pi=0.2(\mathrm{~Hz})$. The trajectory of the slope angle is generated using the sinusoidal function. The DC motor that adjusts slope angle was controlled by the high gain PD control to this desired trajectory.

The learning started not initially but at $15.0(\mathrm{sec})$ since the balance of the robot was certainly maintained from variable initial conditions. The control torque was calculated every 2 (msec) and the feedback gains or parameters were set as $K_{d}=$ $12, K_{p}=70, K_{f}=2, \Gamma=\operatorname{diag}[5, \cdots, 5]$ and $n=8$. The duration of the experiment was set to 100 (sec).

\section{Results}

During the experiment, the robot kept the balance on the slope stand whose gradient was altered periodically. The profile of the ankle joint torque is depicted in Fig. 5. The similar learning process to the simulation result can be observed: the feedback component is decreasing while the feedforward component is increasing on the contrary without affecting

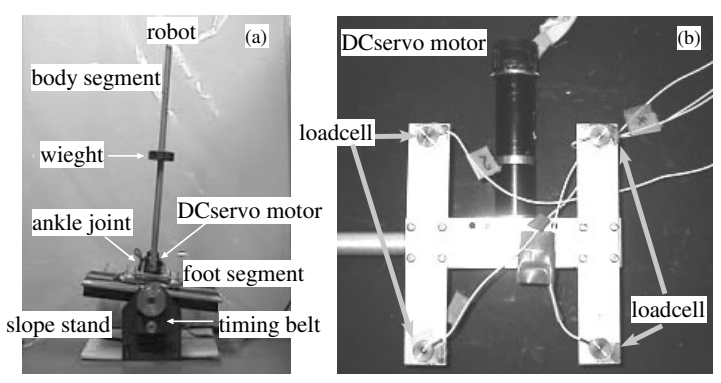

Fig. 4. Robot and slope stand. 


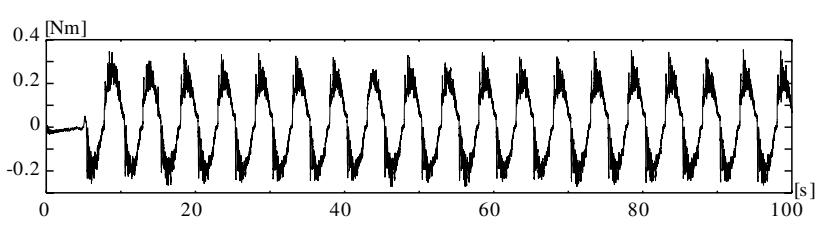

(a) Total torque

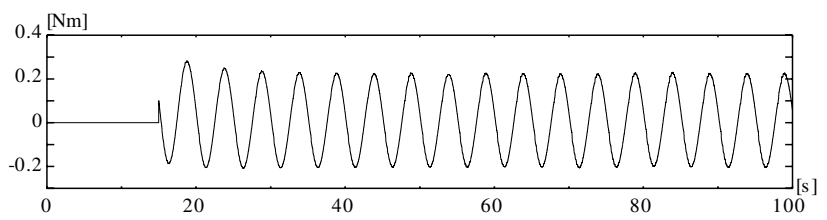

(b) Feedforward component

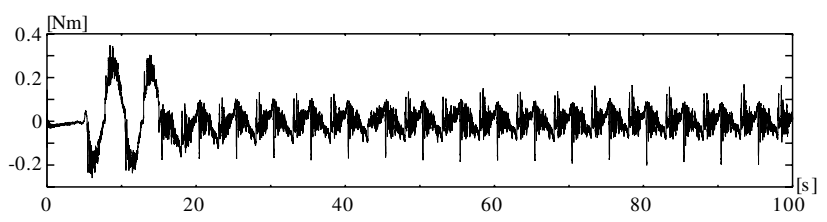

(c) Feedback component

Fig. 5. ankle joint torque at the robot experiment.

the profile of the total torque. This replacement between two torque components indicates that the torque pattern is acquired inside the controller and thus produced in the feedforward manner without feedback information.

\section{DISCUSSION AND CONCLUSION}

In this paper, we took the static balance control as an example to examine the robot's behavior under uncertain environment. Based on the theoretical framework for this simple example, we showed by the practical robot experiments that the torque pattern is gradually produced in the controller without essential feedback information on the ground reaction forces to behave the unknown environmental conditions. To accomplish this learning, the stationarity of environment will be important.

The balance control against external force was reported in [7], and the learning rule where the feedforward torque replaces the feedback ones have already proposed in [8], [6] and so on. However, a new mathematical development is found in our framework at the point that feedback component of the torque includes the information of the force, i.e., ground reaction force. This information is essential to cope with uncertainty in the environmental condition, and thus our balancing control is achievable even on unknown rough terrain. Actually, the unknown external forces will be well simulated to the unknown rough terrain. Instead of the force sensors of ground reaction, an accelerometer and a gyroscope will be effective. However, some careful treatment will be needed since a posture intentionally leaning the body segment is desirable under external force: in this case, the data from gyroscope is not informative because the desired posture is unknown under unknown external force. Our framework in this paper covers such situation thanks to the usage of the ground reaction force information.

In our experimental results, the feedback component does not become zero completely. One possible reason of this is friction or backlash in bevel gear drive at the ankle joint part. Such nonlinear effects will cause the not-smoothing, irregular change of the torque pattern. Especially, the irregularities cannot be learned because of the unpredictability. To cope with such unpredictability, the feedforward information only is not sufficient and thus the feedback of sensory information is also necessary. In addition, the small number of the basis functions of the Fourier series is considered as another reason.

As the future works, we should extend this framework to periodic external force with unknown period, and finally to dynamic walking in uncertain environment. Especially, to apply it to high-DOF robots, further development will be necessary. However, we do not think the essence of the balance control differs so much in the simple model.

\section{REFERENCES}

[1] S. Ito, T. Nishigaki, and H. Kawasaki, "Upright posture stabilization by ground reaction force control," Proc. of the First Int. Symp. on Measurement, Analysis and Modeling of Human Functions, pp. 515-520, 2001.

[2] S. Ito, Y. Aoyama, and H. Kawasaki, "Static balance control and external force estimation using ground reaction forces," Proc. of 2nd Int. Symp. on Adaptative Motion of Animals and Machines, pp. WeP-I-4, 2003.

[3] _ "A static balance control under periodic external force," Proc. of SICE Annual Conference 2003 (CD-ROM), pp. 600-605, 2003.

[4] Y.-G. Ko, J. H. Challis, and K. M. Newell, "Postural coordination patterns as a function of dynamics of support surface," Human movement science, vol. 20, pp. 737-764, 2001.

[5] R. B. McGhee and A. A. Frank, "On the stability properties of quadruped creeping gaits," Mathematical Biosciences, vol. 3, pp. 331-351, 1968.

[6] W. P. L. J-J. E. Slotine, Applied Nonlinear Control. Prentince Hall, 1991.

[7] A. Takanishi, T. Takeya, H. Karaki, and I. Kato, "A control method for dynamics biped walking under unknown external forces," IEEE Int. workshop on Intelligent Robots and Systems (IROS'90), pp. 795-801, 1990.

[8] H. Gomi and M. Kawato, "Neural network control for a closed-loop system using feedback error learning," Neural Networks, vol. 6, pp. 933946, 1993. 